\title{
Análisis de procesos cognitivos en el comportamiento de estudiantes de último curso de bachillerato y primer curso de carrera involucrados en tareas de clasificación, cálculo, memorización y categorización de información
}

\author{
Juan Antonio Martínez Comeche \\ Jorge Horcas Pulido \\ Zuriñe Piña Landaburu *
}

Artículo recibido: 9 de agosto de 2013. Artículo aceptado: 27 de febrero de 2014.

\section{RESUMEN}

Análisis de los procesos cognitivos que subyacen a las tareas de clasificación, cálculo, memorización y categorización de información. Los estudios empíricos sobre el comportamiento de los usuarios involucrados en tareas de búsqueda de información implican normalmente el análisis de tareas complejas. Como resultado, solamente pueden deducirse estrategias de comportamiento de carácter general. A fin de analizar más detalladamente el comportamiento de los participantes, se propusieron a los estudiantes tareas más limitadas y se trató luego de descomponer estas tareas en procesos cognitivos. Se utilizaron técnicas complementarias de recopilación de datos: ficheros log, grabación de las sesiones y entrevistas en profundidad. Con el fin de analizar en profundidad el comportamiento de los participantes, se utilizó la Teoría Fundamentada

Los tres autores pertenecen a la Universidad Complutense de Madrid, España. juan.comeche@pdi.ucm.es 
como método de análisis de datos. El análisis identificó varios procesos cognitivos como los principales componentes de las tareas de clasificación, cálculo, memorización y categorización de información. Estos procesos cognitivos configuran el núcleo del comportamiento de los participantes.

Palabras clave: Comportamiento informacional; Búsqueda y recuperación de información; Procesos cognitivos; Tareas informativas.

\section{Abstract}

Analysis of cognitive processes in the behavior of high school seniors and first year undergrads executing task associates with classification, calculation, memorization and categorization of information

Juan-Antonio Martinez-Comeche, Jorge Horcas-Pulido and Zuriñe Piña-Landaburu

This paper offers and analysis of the cognitive processes entailed in classification, mathematical calculation, memorization and categorization tasks. Empirical studies of information seeking behaviors commonly ask participants to do complex tasks. As a result, only general cognitive strategies may be deduced about information seeking behaviors. To these question in greater depth, this research breaks down the information behavior into atomized tasks reflecting cognitive processes. Three data collection techniques were employed: log files, video recording and in-depth interviews. Grounded Theory was employed as a data analysis method, allowing researchers to discern the behaviors of subjects. The study uncovered several cognitive processes as the main components of simple information-seeking tasks. Moreover, these cognitive processes constitute the core of the subjects' behaviors of the participants. This study supports the furtherance theoretical models of behaviors for several information-seeking tasks, understood as chain of cognitive processes. Some of these cognitive processes apparently are common to entirely distinct tasks, such as classification, memorization and categorization.

Keywords: Information Behavior; Information 


\section{INTRODUCCIÓN}

$U^{n}$ no de los aspectos más relevantes que aborda la Documentación es el relativo al Comportamiento Informacional, que puede concebirse como el área de las Ciencias de la Documentación dedicada al análisis de la forma en que las personas necesitan, buscan, gestionan, difunden y usan la información en distintos contextos (Fisher, Erdelez \& McKechnie, 2005). Esta amplitud en los aspectos que son de interés para los estudiosos del comportamiento informacional ha sido expandida por Tom Wilson (1997), uno de sus máximos representantes, al poner la totalidad del comportamiento humano en relación con los recursos y canales de información, incluida la búsqueda de información, tanto activa como pasiva, y el uso de ésta.

Comprender el comportamiento de los usuarios involucrados en tareas informativas y, en general, cómo interactúan las personas con la información ha sido tradicionalmente un área de investigación de interés para nuestros profesionales. En un principio las investigaciones sobre Comportamiento Informacional se realizaron desde la perspectiva del sistema, incidiendo principalmente en la utilización de determinados recursos de información, como libros o bases de datos, y en el análisis de ciertos procesos vinculados al uso de la información, como el préstamo (González-Teruel, 2011). Fue posteriormente, a partir de la década de 1980 (Bates, 2010), cuando se estableció un giro hacia una aproximación más centrada en el usuario (Wilson, 2000). Aunque han sido muchos los modelos que desde entonces se han propuesto para abordar cuestiones relativas al Comportamiento Informacional (Information Behavior) siguiendo este cambio de paradigma (Wilson, 1999), todos ellos centran su interés en tratar de comprender los modos de actuar de grupos de usuarios en diversos contextos y ambientes. Dicha comprensión se realiza expresamente desde un enfoque cognitivo en la mayoría de ellos, desde Belkin y Wilson (Belkin, 1990; Wilson, 1981) hasta las propuestas más recientes (Spink \& Cole, 2005).

\section{Marco teóRICO}

El enfoque cognitivo del que nosotros partimos (Ingwersen, 2001), común a la generalidad de los autores que afrontan el estudio del comportamiento informacional desde la perspectiva del usuario, incide específicamente en la observación de los procesos intelectuales y el pensamiento interno de los usuarios involucrados en tareas informativas. De esta forma, no importa tanto el comportamiento observado, lo que interesa principalmente es lo que el 
usuario ha pensado y los factores que lo han llevado a actuar de esa manera, partiendo siempre de su estructura de conocimiento. Estructura que involucra todo un sistema de categorías, conceptos y las relaciones existentes entre ellos, de manera que en su conjunto forjan un modelo del entorno y del mundo (Ingwersen, 1982).

Otra de las características del enfoque centrado en el usuario consiste en afrontar la investigación de las tareas informativas desde una perspectiva integral, pues cualquier factor o condicionante puede en un momento dado explicar el modo de actuar del usuario (González-Teruel, 2005). De ahí que los modelos sobre el comportamiento de los usuarios en tareas de búsqueda y recuperación de información describan estas actividades como procesos complejos. Ello no obsta para que la intención primordial del análisis consista en descubrir las principales etapas o acciones de que constan tales procesos complejos de índole informativa, como veremos a continuación al comentar los modelos más destacados desde esta perspectiva.

Así, Nicholas J. Belkin parte de un Estado Anómalo de Conocimiento en el usuario (Anomalous State of Knowledge o ASK) y la necesidad de superar dicho estado. Para ello el usuario inicia un proceso dinámico que involucra sucesivas acciones (búsqueda, selección, reconocimiento...) por las que va adquiriendo cada vez un mayor nivel de experiencia y pericia sobre su propio estado y su posible solución (Belkin, 1993). Por su parte, las investigaciones de Carol Kuhlthau la llevan a distinguir seis etapas en todo proceso de búsqueda de información, ahora con la incertidumbre y la confusión internas del usuario como factores inherentes a todo el proceso: inicios, selección, exploración, formulación, colección y presentación (Kuhlthau, 2004). Brenda Dervin desarrolla su modelo en torno al concepto de "grieta o brecha en el conocimiento", la cual se produce cuando el usuario detecta una falta de comprensión de los datos o de la realidad circundante. Se inicia entonces un proceso para "dotar de sentido" (Sensemaking) a esos datos o realidad inconsistente con el modelo mental del usuario mediante la modificación o alteración del modelo del entorno o del mundo (Dervin \& Nilan, 1986).

En este breve repaso por los modelos centrados en el usuario debemos destacar a Charles Cole, por su relación con el trabajo presentado aquí. En uno de sus artículos (Cole, 1997) intenta identificar patrones comunes en la actividad cognitiva de los individuos que afrontan tareas de búsqueda de información. Mediante el empleo de la Teoría Fundamentada (Grounded Theory), a través de entrevistas a una muestra de 45 alumnos de doctorado en Humanidades, este autor concluye proponiendo un modelo de búsqueda de información que consta de cinco fases: inicios, representación, corroboración, finalización y repercusión. Durante estas fases los sujetos van modi- 
ficando sus estructuras cognitivas y adquiriendo una mayor conciencia de su comprensión del entorno.

Los estudios sobre el comportamiento de los usuarios involucrados en tareas informativas implican normalmente el análisis de tareas de búsqueda de carácter muy general (Fidel et al., 1999; Kim, 2007), que tratan de respetar o de reproducir un escenario lo más similar posible al mundo real. En consecuencia, como hemos visto, se han podido identificar fases, etapas o acciones igualmente de carácter general, relegando el análisis de las acciones concretas seguidas en cada una de dichas fases (Thatcher, 2006).

A fin de lograr un conocimiento más detallado del comportamiento de los seres humanos involucrados en procesos de búsqueda de información, nos proponemos en este estudio considerar tareas parciales habituales en tales procesos, pero de carácter más restringido, tal como hace, por ejemplo, la Teoría Sistémica-Estructural de la Actividad (Systemic-Structural Theory of Activity o SSTA), basada en la Teoría de la Actividad (Activity Theory o AT) (Nardi, 1996). Este enfoque concibe una actividad "as a goal directed system in which cognition, behaviour and motivation are integrated and organized by goals and the mechanisms of self-regulation" (Bedny; Seglin \& Meister, 2000: 168), por lo que podría englobar todos los procesos de búsqueda de información que nos interesan en nuestra área de conocimiento. La SSTA, por su parte, aborda el análisis en profundidad de las actividades descomponiéndolas en una secuencia de elementos menores (tareas, acciones y operaciones), organizada jerárquicamente, las cuales pueden ser sucesivamente subdivididas tanto como sea preciso (Von Brevern \& Synytsya, 2006; Bedny $\&$ Harris, 2005).

En resumen, este estudio se centra en el comportamiento de estudiantes involucrados en cuatro actividades que habitualmente forman parte de las tareas de búsqueda y recuperación de información de carácter general consideradas en la literatura sobre comportamiento informacional. No atendemos, pues, al proceso de búsqueda en su integridad, sino a algunas de las subtareas de que suelen constar dichos procesos. Nuestro principal objetivo es comprobar si es posible discernir, en cada una de estas tareas parciales, acciones o procesos cognitivos más simples, y cuál es la naturaleza de dichos procesos cognitivos, siguiendo un análisis semejante al abordado con las actividades de búsqueda de información de carácter general.

1 “[...] un sistema dirigido a una meta, en donde la cognición, el comportamiento y la motivación son integrados y organizados por las metas y los mecanismos de autorregulación.” (N. del E.) 


\section{Las tareas}

En la elección de las tareas se ha tenido en cuenta el cumplimiento de las dos condiciones esenciales siguientes:

- Debe implicar la realización, por parte del sujeto, de un proceso intelectual. De esta forma se garantiza la concurrencia y puesta en juego de conceptos (un estado de conocimiento), el cumplimiento de un objetivo y la superación de las carencias informativas (estados anómalos, brechas...) que puedan surgir hasta lograr dicho objetivo. Ello fuerza la necesaria intervención de los consiguientes procesos cognitivos cuyo análisis se desea abordar.

- Debe ser parte habitual y especialmente frecuente en actividades de búsqueda de información de carácter general, e igualmente común en múltiples actividades cotidianas. De esta forma se garantiza en mayor medida la generalidad de los resultados obtenidos.

De las distintas posibilidades iniciales consideradas, se eligieron finalmente las tareas enumeradas a continuación, por ser especialmente frecuentes en las actividades de búsqueda y recuperación de información:

- Clasificación. Bajo esta denominación aludimos a la tarea de buscar información sobre un tema teniendo que averiguar bajo qué apartado o clase se habrá organizado. Por ejemplo, si busco información sobre ciertos estudios en la página web de una universidad, dicha información ha podido ser clasificada dentro del apartado "Facultades" o dentro del apartado "Estudios".

- Concepto matemático y cálculo elemental. Tarea consistente en seleccionar un número, de entre varios posibles, que se ajuste a un concepto matemático simple, para lo que se deben efectuar cálculos numéricos sencillos. Cuando realizamos una reserva de habitación y manutención en un hotel, por ejemplo, tenemos que calcular el gasto total que supondrá para toda la familia esta reserva teniendo en cuenta el número de miembros y los días de estancia, lo que implica sumas y multiplicaciones básicas. A su vez, el gasto total calculado debe ajustarse al presupuesto familiar, por lo que debe ser menor que una cierta cantidad prefijada de antemano.

- Memorización. Tarea consistente en memorizar datos para poder continuar con el proceso informativo iniciado. Esta tarea se produce, por ejemplo, cuando debemos memorizar varios nombres de lugares pre- 
feridos para veranear y a continuación buscar hoteles de determinado precio en la zona donde se encuentran los lugares elegidos, para ver si coinciden el lugar y el hotel seleccionados.

- Categorización. Bajo esta denominación aludimos a la tarea de adscribir datos a categorías fijadas de antemano. Esta situación se produce, por ejemplo, cuando rellenamos formularios por Internet: si no hay una categoría destinada específicamente a cierta información dudamos entre las opciones disponibles. Es una tarea complementaria de la clasificación: en ella se trata de que el usuario averigüe la categoría elegida por el gestor de la información, mientras que en la tarea de categorización se trata de que el usuario elija la categoría que considera más adecuada.

\section{Metodología}

Participaron 40 voluntarios, en su inmensa mayoría entre los 18 y los 22 años; $57 \%$ correspondió a mujeres y $43 \%$ a hombres ( 23 mujeres frente a 17 hombres). Los sujetos fueron escogidos entre el último año de bachillerato y el primer año de carrera, todos ellos con especialidad en Humanidades y Ciencias Sociales, y ninguno especializado en Ciencias Experimentales.

La elección de los sujetos y su perfil formativo concuerda con las características de la población empleada en la mayor parte de los estudios sobre comportamiento informacional basados en la perspectiva del usuario: estudiantes de diferentes grados (con una preponderancia de los niveles de posgrado y doctorado), científicos, investigadores y usuarios de servicios de información, y ocasionalmente empleados de empresas.

En cuanto al número de sujetos que intervienen en este tipo de estudios, en una cantidad significativa de ellos las muestras no superan los 50 participantes. Así, Kuhlthau (1983) empleó 26 alumnos de último curso de bachillerato en su tesis doctoral; Cole (1997) se sirvió de 45 alumnos de doctorado para elaborar su modelo; en estudios más recientes, el de Gómez Restrepo (2012) por ejemplo, se utilizó una muestra de 35 estudiantes de una población de 116 alumnos de tesis de maestría y doctorado de El Colegio de México; de igual forma, Xuemei Ge (2010) entrevistó a 30 profesores y doctorandos de Humanidades y Ciencias Sociales de la Universidad Estatal de Tennessee para conocer cómo influyen los recursos electrónicos en los procesos de búsqueda de información en las áreas de Ciencias Sociales y Humanidades.

La población objeto de estudio se eligió entre los alumnos de último curso de bachillerato y primer curso de carrera por formar parte de la deno- 
minada "Generación Google" (nacidos después de 1993), cuyos hábitos y comportamiento informativo han sido analizados ampliamente en el Informe CIBER (British Library \& JISC, 2008). Ello nos garantiza que todos los participantes están acostumbrados a utilizar Internet para acceder al conocimiento y que suelen emplear los motores de búsqueda generalistas para localizar información. De hecho, sólo un $2 \%$ aproximadamente inicia la búsqueda de información a través de la página web de una biblioteca, como han corroborado los estudios realizados entre los estudiantes de la Universidad de Alicante (Alemany \& Candela, 2011). Se ha constatado igualmente un comportamiento deficiente común a este sector de la población relacionado con la falta de reflexión sobre sus necesidades informativas y con la falta de enjuiciamiento crítico sobre los recursos localizados en la Web, situación que las bibliotecas universitarias tratan de paliar (Gómez Hernández, 2010). Al mismo tiempo, nos aseguramos previamente de que los participantes no tuviesen conocimientos específicos ni de Economía ni de Biblioteconomía, pues la prueba de memorización incluía textos de estas materias. De esta forma, la uniformidad de conocimientos, hábitos y comportamientos de la población objeto de estudio facilita la obtención de resultados generalizables aunque la muestra no sea especialmente amplia.

Los participantes se dividieron en dos cohortes, una primera con 24 sujetos pertenecientes al curso lectivo 2012-2013 y una segunda con 16 sujetos del curso 2013-2014. Se les dieron cuatro tareas a realizar. Cada sujeto de la primera cohorte realizó las cuatro tareas en un orden diferente. Para evitar el efecto distorsionador relacionado con el orden con que los 16 sujetos de la segunda cohorte realizaron las tareas, se seleccionaron al azar 16 secuencias de tareas de entre las 24 posibles (León \& Montero, 1999).

Las tareas encomendadas fueron las siguientes y tuvieron un límite temporal de 6 minutos para la realización de cada una de ellas:

- Clasificación. Se les explicaron a los sujetos las principales características y categorías del directorio Buscopio en español (www.buscopio. net/esp/), a fin de que no tuviesen problemas idiomáticos. Se les pidió que, sin utilizar ningún tipo de buscador, exclusivamente navegando dentro del directorio, encontrasen el tema "libros electrónicos" e indicasen la ruta donde se encuentra clasificado.

- Concepto matemático y cálculo elemental. El sujeto tenía que realizar una serie de búsquedas de datos numéricos específicos en una página web (memorizada en el navegador de antemano) y a raíz de esos datos, realizar dos tareas principalmente: en primer lugar, seleccionar un número de entre varios basándose en un concepto matemático simple 
(elegir el mayor que fuese número primo); en segundo lugar, efectuar operaciones matemáticas sencillas con algunos números (sumas y multiplicaciones). El sujeto podía utilizar una hoja de borrador, cualquier programa del ordenador (una calculadora, por ejemplo) o cualquier recurso de Internet en caso de duda.

- Memorización. El sujeto tenía que memorizar tres textos (2 minutos por texto) que van aumentando progresivamente en longitud y dificultad (considerando el nivel de dificultad por el desconocimiento del tema): una sucinta biografía de un futbolista español, datos económicos sobre España, Biblioteconomía y Documentación. Posteriormente el participante debía responder dos preguntas sobre datos concretos de cada texto.

- Categorización. El sujeto tenía que relacionar cada una de las seis páginas web (memorizadas en el navegador de antemano) con una única categoría sobre seis categorías posibles, de manera que solamente podía asignar cada página web una única vez a una categoría. El sujeto podía consultar cualquier recurso de Internet en caso de duda.

Esta versión definitiva de las tareas fue adoptada tras realizar un pretest con seis sujetos que nos permitiese no sólo comprobar el correcto diseño de las tareas, sino también el correcto funcionamiento de los programas y de la cámara, además de la adecuada recolección de datos. En este paso se decidieron cuatro cambios en relación al diseño inicial de las tareas:

- Se había fijado un periodo de 4 minutos para la realización de cada tarea. En la versión definitiva se aumentó la duración de cada tarea a 6 minutos, de manera que una mayoría de los participantes pudiese concluir todas las tareas.

- La tarea de memorización constaba de un único texto. Finalmente se prefirió diseñar esta tarea con tres textos de longitud y dificultad variables, con el objeto de incluir también estos factores en el estudio.

- La tarea de categorización no incluía explícitamente la obligatoriedad de adscribir cada página web a una única categoría. La versión definitiva de esta tarea incluye dicha obligatoriedad, para evitar comportamientos dispares en los sujetos.

- Se concibió entrevistar una sola vez a cada participante tras realizar todas las tareas. Finalmente cada participante fue entrevistado después de cada tarea, con la intención de que recordase perfectamente los pensamientos y el porqué del procedimiento seguido en cada una de las tareas encomendadas. 
Se emplearon tres métodos simultáneos de recopilación de datos: archivos $\log$, entrevistas en profundidad no estructuradas (Valles, 1997) y grabación en video de todas las sesiones, a fin de observar y registrar los comportamientos de los participantes. De este modo pudimos recopilar información exhaustiva sobre la actividad de cada sujeto en el ordenador (programas empleados, búsquedas realizadas y páginas web visitadas). Las entrevistas en profundidad no estructuradas se realizaron justo después de cada tarea, en un formato totalmente abierto, de manera que los participantes pudiesen explicar perfectamente cómo habían actuado y por qué. Las ideas y acciones fueron surgiendo a lo largo de las conversaciones y buscaban limitar nuestras intervenciones a incitar a los participantes para que ahondasen en sus pensamientos, actitudes y comportamientos (el guión de la entrevista en profundidad puede consultarse en el Anexo).

Todas las entrevistas fueron analizadas y codificadas empleando la Teoría Fundamentada (Grounded Theory) (Strauss \& Corbin, 1998), aunque redujimos el proceso de análisis a sus dos fases principales (Charmaz, 2006: 46), pues los datos recopilados presentaron mayor semejanza y coherencia de la esperada inicialmente. De hecho, se alcanzó la saturación de categorías en la entrevista 18 (Glaser \& Strauss, 1967). Las dos fases seguidas son las siguientes:

- Una fase primera de selección de fragmentos significativos que eliminaba redundancias o comentarios personales ajenos a los objetivos del estudio (opiniones sobre las tareas, comentarios sobre sus circunstancias personales, incluso en ocasiones sus dudas sobre la correcta realización de las tareas), y una categorización inicial mediante la comparación constante y la denominación común a los fragmentos que mostraran ideas semejantes (correspondientes, en líneas generales, a la codificación abierta y a la codificación enfocada).

- Una segunda fase en la que las categorías más significativas y frecuentes se integraban y se organizaban en un modelo de cada tarea analizada (englobando tanto la codificación axial como la codificación teórica).

\section{Resultados}

En cuanto a la tarea de localización del tema "libros electrónicos" en el directorio Buscopio (tarea de clasificación), cabe destacar que las categorías principales cuya relación con "libros electrónicos" se ha percibido como más 
probable, considerando el número de sujetos que las eligieron en primera o segunda opción, son las siguientes:

- Informática. El motivo de la relación es doble: bien porque el libro electrónico es percibido como un dispositivo electrónico, como lo es una computadora (y está vinculado al hardware), o bien porque el libro electrónico es considerado como un formato de datos, de carácter digital (y por tanto vinculado al software).

- Aficiones-ocio. El libro electrónico se observa como un aparato de ocio que se usa para la lectura. A su vez, la lectura se considera como una de las principales aficiones posibles.

Los sujetos han buscado "libros electrónicos" en otras dos categorías principalmente, aunque a gran distancia de las anteriores por número de intentos:

- Medios de comunicación. En este caso, dos han sido los razonamientos realizados por los participantes para considerar posible la inclusión de "libros electrónicos" en ella: como hay periódicos y revistas electrónicos (un formato más tecnológico), también podían haberse englobado en esta categoría los libros electrónicos. El segundo razonamiento sería el siguiente: una de las subcategorías visibles bajo esta categoría principal es "e-zine", por lo que era posible una relación entre e-zines y e-books.

- Internet. El razonamiento también es doble: bien porque Internet está vinculado con lo electrónico, bien porque algunos libros electrónicos tienen capacidad de tener conexión con Internet.

Los conceptos finales que el análisis de la tarea de localización de un tema en un directorio nos permite considerar como comunes y significativos, desde un enfoque cognitivo, fueron los siguientes. Se incluyen ejemplos extraídos de las entrevistas para ilustrarlos (S_x indica el número de sujeto):

- Exploración del sistema de clasificación.

"Cuando he entrado en Buscopio, he visto todas las actividades que había." (S_15)

"Leer primero los títulos grandes [las categorías principales], y luego los pequeños [las subcategorías].” (S_35) 
- Asociación del tópico buscado a categorías.

"He intentado pensar en qué campo podía estar incluido el libro electrónico.” (S_10)

"Después de pensar todas y ver un poco y decir... puede ir por aquí." (S_37)

- Ordenación de categorías por intensidad de la asociación.

"De los puntos que he visto al principio, los que tenían más probabilidades de estar ahí." (S_2)

"He ido descartando los que no podían ser, como por ejemplo los Deportes, la Educación, y he ido mirando los que tienen relación, como la Informática e Internet." (S_33)

- Localización del tópico buscado en las categorías.

"Primero me metí en Informática e Internet, y busqué ahí todo lo que pude.” (S_16)

"Luego también he estado en Educación, por si acaso había apuntes y libros." (S_34)

En relación con la segunda tarea, dos eran las cuestiones de carácter matemático encargadas a los sujetos: por una parte, la realización de cálculos matemáticos sencillos (sumas y multiplicaciones); por otra, la selección de números basada en conceptos matemáticos sencillos (elegir el mayor número primo de entre varios). Cabe destacar que los participantes se han ayudado fundamentalmente de dos recursos para resolver sus dudas sobre las cuestiones matemáticas planteadas:

- Una calculadora, en relación con la tarea de cálculo de operaciones sencillas.

- Un buscador web generalista, en relación con la tarea de utilización de un concepto matemático sencillo (número primo).

La calculadora ha sido utilizada mayoritariamente cuando el sujeto tenía que multiplicar dos números y cada uno de ellos tenía dos cifras o más. En caso contrario (multiplicación de dos números, uno de los cuales es de una cifra, o bien la suma de dos números, sin importar el número de cifras), los participantes realizaron el cálculo de memoria o en un papel y sólo algunos 
de ellos usaron la calculadora para asegurarse de que el resultado obtenido era correcto.

El buscador web generalista empleado por todos los sujetos para resolver dudas sobre los números primos fue Google, excepto en un caso que se utilizó Bing. La frase de búsqueda más habitual fue "número/s primo/s", y la página más visitada fue http://es.wikipedia.org/wiki/Número_primo (la primera en la página de resultados del buscador).

Como estaba previsto, el proceso cognitivo seguido en los cálculos matemáticos sencillos no aporta grandes resultados, pues se basa esencialmente en el recuerdo de las tablas de sumar, de las tablas de multiplicar y de los procesos iterativos de suma y multiplicación. Lo más destacable ya ha sido comentado previamente en cuanto a que los sujetos emplearon recursos de apoyo (una hoja de papel o una calculadora) dependiendo de las características de los números con los que se realizó el cálculo.

La utilización de un concepto matemático (número primo, en este caso) permite obtener resultados de mayor alcance, pues la formación matemática básica de los sujetos hizo que aproximadamente la mitad de ellos no recordase el concepto matemático propuesto. Los conceptos finales que el análisis de la tarea de selección de números basándose en conceptos matemáticos sencillos (elegir el mayor número primo de entre varios) nos permite considerar comunes y significativos, desde un enfoque cognitivo, fueron los siguientes; para ilustrarlos se incluyen ejemplos extraídos de las entrevistas (S_x indica el número de sujeto):

- Recuerdo del concepto matemático.

"He tenido que pensar primero lo que era número primo porque no me acordaba." (S_1)

"He tenido que buscar en Google qué era un número primo porque lo recordaba, pero no sabía exactamente qué era cien por cien.” (S_27)

- Reconstrucción de la lista de números conforme al concepto matemático.

"Recordaba que el número primo es el que se divide por sí mismo y por la unidad, o algo así, pero no sé si habrá alguien que se sepa la lista de los números primos; yo no me lo sé.” (S_5)

"He buscado la lista de números primos en Internet." (S_34)

- Selección del número ajustado al concepto matemático que verifique otro u otros criterios preestablecidos. 
"Mirando todas las medallas de plata que había a lo largo de todos los países y viendo cuál era el [número] primo y cuál era el mayor." (S_11) "Lo que he hecho ha sido ir bajando y viendo cuáles [países] tenían mayores [número de medallas de plata], y dentro de esos, cuál era el número primo. He mirado así hasta el final, por si había alguno que podía superar al que tenía hasta ese momento.” (S_29)

En relación con la tarea de memorización, se les pidió a los sujetos que efectuasen un proceso de memorización a muy corto plazo con tres textos de distinta longitud y dificultad (por desconocimiento previo del tema). Los resultados obtenidos pueden clasificarse en dos: unos relativos al proceso en sí de memorización y otros relativos al grado de éxito obtenido. Entre los resultados obtenidos en relación al proceso en sí de memorización, destacan los siguientes:

- La tarea en sí de memorización a muy corto plazo no depende de la temática o del tipo de datos que deban procesarse. Los sujetos, casi en su totalidad, reconocen seguir siempre un mismo proceso, independientemente del tipo de texto.

- En caso de seguir procesos distintos dependiendo de la temática, existe un núcleo importante del proceso que es común a cualquier tema. Las diferencias se limitan a la introducción de refuerzos en los casos considerados necesarios.

Con relación al grado de éxito o fracaso en el proceso de memorización, los resultados más destacados son los siguientes:

- El resultado de la memorización a muy corto plazo depende del nivel de concentración de los datos que deben ser memorizados. Cuanto mayor es dicha concentración, más difícil resulta memorizar y el resultado obtenido es peor.

- La tarea de memorización a muy corto plazo depende también del grado de estructuración y organización del texto (esto es, si los datos están relacionados entre sí conforme a una lógica expuesta claramente en el texto). Si es posible ir reflexionando y madurando la información recibida conforme se va leyendo el texto, la memorización se facilita. En cambio, datos inconexos entre sí son más difíciles de memorizar y se obtienen resultados peores.

- El éxito en la memorización a muy corto plazo depende del tipo de datos que deban memorizarse, lo que resulta en una tasa de éxito mayor cuando se memorizan ideas que cuando deben memorizarse palabras 
o números concretos. Los sujetos explicaron este hecho afirmando que una idea se puede recrear con palabras propias, siempre que se transmita la idea en sí, de manera que se considera el objetivo de memorización cumplido con muchas más posibilidades de expresión, mientras que los datos específicos (palabras o números) poseen un grado de libertad nulo a la hora de considerar exitoso el proceso.

- El grado de éxito en la tarea de memorización a muy corto plazo depende del nivel de comprensión previo de los términos y conceptos involucrados. El saber reconocer la terminología empleada y el conocimiento previo de los principales conceptos utilizados provocará un nivel de éxito mayor. El desconocimiento de la temática (por el léxico y las ideas transmitidas) provocará una tasa de fracasos mayor en el proceso. En consecuencia, cuando la temática es cotidiana o de actualidad, el éxito es mayor, aunque los datos concretos sean previamente desconocidos por los sujetos.

- Los sujetos concuerdan en que el tiempo concedido para la memorización influye también en el resultado del proceso: cuanto más tiempo para memorizar, mejor será el resultado.

- En cambio, los sujetos coinciden en que la longitud del texto que se debe memorizar no es especialmente relevante, para un nivel dado de comprensión previo de los términos y de los conceptos involucrados.

Los conceptos finales que el análisis de la tarea de memorización nos permite considerar comunes y significativos desde un enfoque cognitivo fueron los siguientes; se ilustran con ejemplos extraídos de las entrevistas (S_x indica el número de sujeto):

- Exploración del texto.

"Primero me lo leo una vez, intento comprenderlo, porque si no, no puedo memorizarlo. Una vez que he comprendido de qué va el texto, lo vuelvo a leer ya quedándome con las cosas." (S_20)

"Primero lo leo completo para tener una idea general." (S_27)

- Asociación de los datos a memorizar entre sí y/o con algún ítem personal (número, fecha, palabra, etc., familiar al sujeto).

"Las fechas las voy relacionando con fechas que yo me sé; por ejemplo, una fecha aproximada a la de mi cumpleaños y es de alguna guerra, pues las relaciono." (S_22) 
"Relacionar, porque a fin de cuentas todo está relacionado. [...] Por ejemplo, sabía que el ISSN estaba relacionado con el ISO [...] Por ejemplo, el 26 lo sé porque lo he relacionado con las temporadas y con los partidos." (S_31)

En cuanto a la tarea de categorización de seis páginas web en seis temas uno a uno, el análisis muestra la relevancia del punto de vista personal a la hora de asignar categorías a las páginas web. Cada sujeto parte de un modelo del mundo propio (entendido como la conjunción de conceptos, criterios, opiniones, conocimientos, recuerdos, experiencias...) en el cual basa esencialmente su elección de categorías. Este modo de proceder resalta especialmente al final del proceso, cuando restan por adjudicar las dos últimas páginas a dos posibles categorías. En caso de dudas sobre el tema que corresponde a cada una de las dos páginas, los sujetos dedican más tiempo a la toma de decisión y acuden a recopilar más información sobre los contenidos de las páginas conflictivas (dónde adscribir las páginas de senderismo y scouts -tiempo libre o deporte- es un ejemplo frecuente). Las propias páginas propuestas a los participantes o los diversos sitios web en español dedicados a estos temas que aparecen en las primeras posiciones de la página de resultados de Google incluyen una determinada opción. Sin embargo, si persiste el conflicto entre su punto de vista personal y la información recopilada, los sujetos tienden a decantarse finalmente por su propio criterio. Así, uno de los sujetos terminaba el razonamiento sobre su elección afirmando: "yo los scouts sí que sé cómo funcionan y sé lo que son” (S_13).

También conviene destacar en esta tarea las secciones o aspectos concretos de los sitios web empleados por los participantes para averiguar su contenido. No se basan en la posible organización o institución que sostiene el sitio web, sino en las siguientes características o secciones -ordenadas por frecuencia-, argumentando que son las que les permiten hacerse con una idea general y rápida del contenido del sitio web:

- Las fotografías, las imágenes y, en general, los elementos visuales incorporados a la página principal del sitio web.

- Sección "Quiénes somos".

- Configuración y diseño de la página principal (si sigue un formato de blog o foro, por ejemplo; en su defecto, la configuración de las distintas secciones en que se divide la página principal y la tipografía empleada).

- Título (elemento <title>) de la página principal del sitio web. 
Los conceptos finales que el análisis de la tarea de categorización nos permite considerar comunes y significativos desde un enfoque cognitivo fueron los siguientes, con ejemplos ilustrativos extraídos de las entrevistas (S_x indica el número de sujeto):

- Exploración de las páginas web y de las categorías disponibles.

"Me he leído las categorías y luego he dado un vistazo a las páginas leyendo un poco por encima." (S_8)

"Primero he leído todas las categorías que teníamos y luego me he ido a dichas páginas para tener una idea." (S_27)

- Asociación de páginas a categorías.

"He vuelto a pasar por todas las páginas y he ido clasificándolas según yo creía que eran." (S_12)

"He intentado un poco ver a lo que podía asociar, así, generalmente." (S_32)

\section{DisCUSIÓN Y CONCLUSIONES}

De los conceptos extraídos tras el análisis de las diversas tareas encomendadas a los participantes, la primera conclusión que se deduce es la presencia de dos procesos cognitivos comunes a tres de las tareas encomendadas a los sujetos. La relación de dichos procesos y su alcance es el siguiente:

- Exploración. Proceso consistente en aprehender las características más relevantes del sistema con el que debe interactuar el sujeto, en función de la tarea abordada y del objetivo final que desea conseguirse. En el caso de las tareas consideradas en este trabajo, la exploración consiste en captar las clases y subclases principales de un sistema de clasificación; o tomar contacto con la temática, el vocabulario y los datos expuestos en los textos a memorizar; o bien observar las categorías y la temática de las páginas web que deben someterse a categorización.

- Asociación. Proceso consistente en relacionar ítems, pertenecientes o no a grupos o clases distintos, mediante la estimación del grado en que diversos atributos, rasgos o propiedades los relacionan o están presentes simultáneamente en dichos ítems. En el caso de las tareas consideradas en este trabajo, la asociación consiste en sopesar bajo qué cate- 
gorías de un buscador puede haberse incluido el libro electrónico en función de diversas facetas posibles suyas (formato, uso...) conforme al conocimiento del participante; u observar la relación existente entre los diversos datos expuestos en cada uno de los textos a memorizar, reconstruyendo la estructura de conocimiento subyacente en el texto, o ayudarse de ciertos datos de la propia estructura de conocimiento del participante para facilitar la memorización de los datos del texto; o bien valorar qué propiedad o rasgo de cada página web considerar, conforme a la propia estructura de conocimiento (incluyendo criterios, opiniones, vivencias...), en el momento de adscribirla a una categoría fijada de antemano.

Como vemos, este último proceso cognitivo es nuclear en las tres tareas de clasificación, memorización y categorización. Y en las tres tareas, componentes habituales en las actividades de búsqueda y recuperación de información, cobra especial protagonismo la estructura de conocimiento del participante, como hemos señalado. Este hecho corrobora la imprescindible consideración de las peculiaridades cognitivas del usuario en el diseño de los sistemas de búsqueda y recuperación de información si deseamos que alcancen un grado elevado de eficacia. Aspecto destacado especialmente por investigadores como Belkin (Belkin, Oddy \& Brooks, 1982), pero compartido por todos los investigadores en Comportamiento Informacional. Conforme a los resultados obtenidos en este trabajo, podemos considerar el proceso cognitivo de asociación como una de las tareas, dentro de las actividades de búsqueda y recuperación, en las que cobra especial relevancia dicha estructura de conocimiento.

A estos procesos cognitivos se añaden otros cuatro, no tan nucleares por cuanto aparecen en una sola tarea, cuya relación y alcance son los siguientes:

- Ordenación. Proceso consistente en priorizar ítems en un cierto conjunto o grupo en función de una o varias propiedades, atributos o rasgos. En la tarea de clasificación se ordenan las categorías de Buscopio por la mayor relación, a priori, con el tópico "libros electrónicos".

- Localización. Proceso consistente en confirmar o negar la presencia de un ítem en cierto apartado, grupo o clase. En la tarea de clasificación se busca el tópico "libros electrónicos" en diversas categorías de Buscopio.

- Recuerdo. Proceso consistente en traer a un primer plano de la memoria una parte de la propia estructura de conocimiento. En la tarea del concepto matemático se debía recordar el concepto de número primo. 
- Reconstrucción. Proceso en ocasiones complementario del anterior, consistente en rehacer o en recomponer parte de la estructura de conocimiento olvidada. En la tarea del concepto matemático se debía reconstruir la lista de los primeros números primos.

Conforme a estos procesos cognitivos descritos, se concluye que la tarea de utilización de un concepto matemático es la más peculiar de las cuatro consideradas, pues al contrario que las tres restantes ninguno de los procesos cognitivos de los que se compone surgen en el resto de tareas. Sin duda, este resultado abre una vía complementaria de investigación en el futuro próximo, que trate de comprobar el grado de excepcionalidad de estos procesos comparando esta tarea con otras más semejantes, en principio, a ella.

De la misma manera que las investigaciones consideradas en el marco teórico han originado modelos donde se descompone la tarea general de búsqueda de información en función de los procesos esenciales detectados en el análisis, uno de los objetivos del presente trabajo consiste en examinar la posibilidad de desarrollar un modelo teórico de estas tareas en función de los procesos cognitivos que las conforman.

En relación con la tarea de clasificación, el orden en que suelen ejecutarse estos procesos vistos no siempre es el mismo. Hay sujetos que prefieren entrar directamente en la que consideran la categoría más probable, y solamente al comprobar que no está allí realizan la exploración de la segunda posible categoría donde localizar el tema "libros electrónicos", y así sucesivamente. Otros, en cambio, son más sistemáticos, y optan por ordenar varias posibles categorías antes de entrar en ninguna de ellas. Ahora bien, incluso los primeros sujetos, cuando afirman entrar en la opción más probable es porque, aún no reconociéndolo, han tenido que realizar la tarea de exploración de las opciones posibles y ordenar dichas opciones para entrar en la primera elegida, pues no se decidieron al azar.

En consecuencia, concluimos que es posible establecer un orden en los procesos cognitivos enumerados anteriormente y establecer así un modelo teórico de comportamiento en relación a la tarea de clasificación, que puede resumirse en el esquema siguiente: 


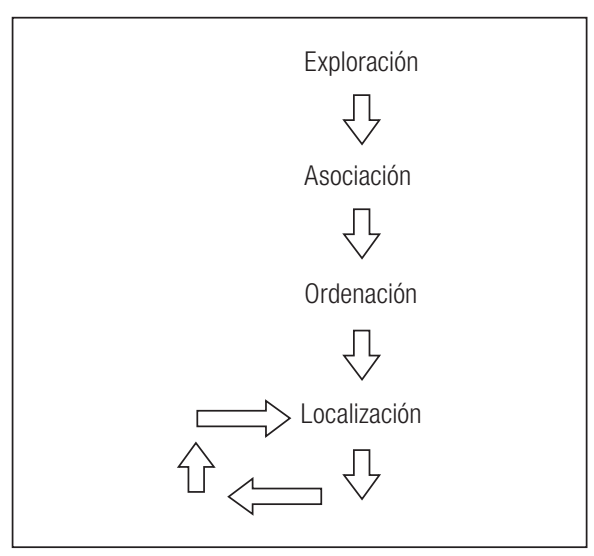

Figura 1. Modelo de la tarea de clasificación

Acerca de la tarea de utilización de un concepto matemático sencillo (elegir el mayor número primo de entre varios), varios aspectos deben ser resaltados. En primer lugar, no todos los sujetos realizan estos dos procesos. En ocasiones algún participante se salta el proceso de recuerdo del concepto y afronta directamente el de reconstrucción de la lista de números, dado que para el objetivo final ese proceso de recuerdo no es imprescindible. De hecho, algunos sujetos buscaron directamente una lista de números primos. Pero si el sujeto decidió calcular uno a uno los pocos números que necesitaba de la lista, tuvo necesidad de recordar el concepto matemático. Solamente cuando el sujeto decide alcanzar su objetivo empleando otra estrategia (preguntando a un amigo, yendo a la biblioteca o, como en este caso, buscando la lista en la Web) es posible eliminar el primer proceso de recuerdo. Comentaremos esta posibilidad a continuación.

Un hecho relevante que ha surgido en la realización de esta tarea es que estos procesos cognitivos de recuerdo y reconstrucción pueden desembocar en tareas sustitutorias de búsqueda y recuperación de información. Es más, en este caso el primer proceso de recuerdo puede desaparecer, como se mencionó anteriormente.

Si el sujeto que ha iniciado un proceso cognitivo de recuerdo no puede finalmente alcanzar su objetivo de recordar el concepto matemático que necesita, iniciará una tarea de búsqueda y recuperación de información con el mismo objetivo final (conocer qué significa el concepto matemático). De igual forma, si un sujeto debe afrontar un proceso cognitivo de reconstrucción y se ve incapaz, o le resulta pesado o complejo dicho proceso, puede optar por efectuar una tarea supletoria de búsqueda y recuperación de información para conseguir el objetivo propuesto (una lista de números primos, en este caso). 
Concluimos, pues, que es posible establecer un orden en los procesos cognitivos citados anteriormente, siempre que surjan ambos. El establecimiento de un modelo teórico de comportamiento en relación a la tarea de utilización de un concepto matemático queda supeditado, pues, a estas dos peculiaridades:

- En primer lugar, cualquiera de los dos procesos cognitivos (recuerdo y reconstrucción) es de aparición optativa. Solamente cuando surgen ambos se puede establecer un orden entre ellos.

- En segundo lugar, cualquiera de los dos procesos cognitivos puede originar una actividad supletoria de búsqueda y recuperación de información.

Con estas salvedades, el modelo correspondiente a la utilización de un concepto matemático puede resumirse en el esquema siguiente:

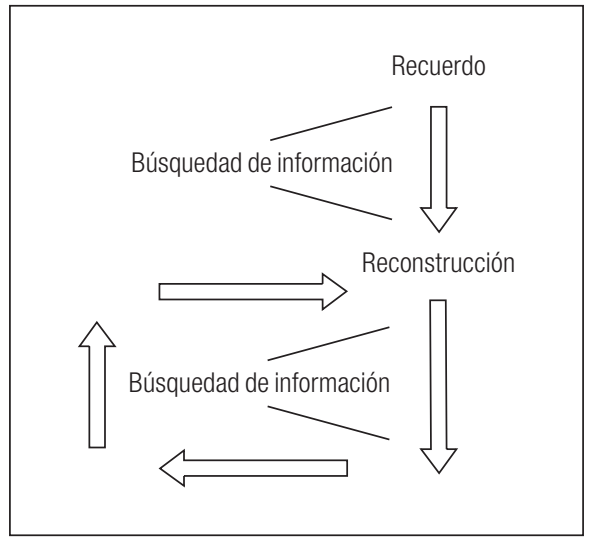

Figura 2. Modelo de la tarea del concepto matemático

En relación con la tarea de memorización concluimos que, en la población observada, las tareas de tal índole constan de dos procesos cognitivos realizados en un proceso iterativo de lecturas sucesivas del texto a memorizar: exploración y asociación.

En ocasiones, estos dos procesos se realizan simultáneamente. Algunos sujetos comentaron que ya en la primera lectura exploratoria aprovechan normalmente para iniciar el proceso de asociación de los datos del texto entre sí (considerándolo subyacente a la comprensión) o de algún dato del texto con otro dato personal. Pero ello no evita que los dos procesos se produzcan en todo proceso de memorización. En consecuencia, concluimos que es posible establecer un orden en los procesos cognitivos enumerados anteriormen- 
te y, por tanto, establecer un modelo teórico de comportamiento en relación a la tarea de memorización que puede resumirse en el esquema siguiente:

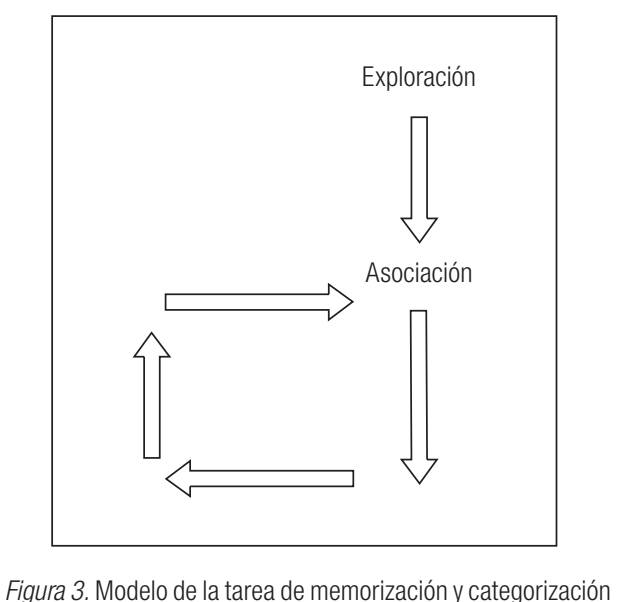

Como hemos visto anteriormente, estos mismos procesos cognitivos básicos (exploración y asociación) son también los que surgen en el análisis de la tarea de categorización, última que fue propuesta a los participantes. De igual manera, aunque en ocasiones en el proceso de exploración inicial los sujetos asocian ya alguna página web con su correspondiente categoría, es claro que dicha relación sólo podría establecerse si se conocen previamente las posibilidades existentes. En consecuencia, se deduce que podemos establecer un modelo teórico de comportamiento en relación con la tarea de categorización que contendría los mismos procesos cognitivos y en el mismo orden que la tarea de memorización, tal como refleja la Figura 3.

De lo expuesto hasta aquí conviene destacar como conclusión final que los procesos cognitivos elementales descubiertos son compartidos por tareas aparentemente muy distintas entre sí, como pueden ser la memorización y la categorización. En consecuencia, el comportamiento informacional de los seres humanos, descrito en función de tales procesos cognitivos, presenta características comunes independientemente de la tarea específica que afronten.

Este mismo fenómeno puede hacerse extensivo a un proceso final de comprobación de la tarea terminada correctamente, común a todas las tareas incluidas en el presente estudio. Este proceso final de comprobación se materializa, en los esquemas correspondientes a las cuatro tareas, mediante un bucle dedicado específicamente a tal fin. Este resultado, por otra parte, ya se había puesto de manifiesto anteriormente. Bedny \& Harris, al exponer las unidades de toda actividad, afirman que "Actions can be described in terms 
of a recursive loop structure [...]" (2005: 132), ${ }^{2}$ con un paso final dedicado a la evaluación de resultados y la corrección. El estudio llevado a cabo aquí corrobora esta idea puesta de manifiesto anteriormente por la Activity Theory, y por múltiples estudiosos de nuestra área de conocimiento (Bates, 1989; Salton, 1968, por ejemplo).

Por último, cabe decir que si, conforme muestran los datos expuestos aquí, es posible profundizar en el comportamiento informacional de los seres humanos mediante la descripción de tareas más simples, como la clasificación, la categorización, el cálculo y la memorización, utilizando para ello procesos cognitivos más elementales, se abre una vía de investigación inmediata consistente en averiguar las diferencias que han de existir necesariamente entre actividades aparentemente tan dispares como la memorización y la categorización. Si podemos invocar un principio de economía, por otra parte común a tantos fenómenos humanos, incluida la Biblioteconomía y Documentación (Zipf, 1949), para argumentar a favor de la utilización por parte de los seres humanos de los mismos procesos cognitivos en tareas tan distintas, el objetivo final y el resultado es muy distinto en tales tareas, lo que ha de poder ser explicado profundizando aún más en el componente cognitivo y de comportamiento de tales actividades.

\section{Reconocimientos}

Agradecemos la ayuda de los profesores Vellosillo González y Gonzalo Sánchez-Molero, por apoyar institucionalmente esta investigación desde sus cargos en la Facultad de Ciencias de la Documentación de la UCM. Igualmente agradecemos la colaboración de los profesores Da Costa Carballo y Sánchez Vigil por la difusión de este proyecto en sus clases y animar a los alumnos a participar en él. También nuestro agradecimiento a Luis Alberto Allende Álvarez y Almudena Polo del Pozo, Director y Secretaria del Instituto Manuel Azaña de Getafe (Madrid), por poner a nuestra disposición todos los medios necesarios para la realización de las pruebas. Agradecemos especialmente a los alumnos de la Facultad de Ciencias de la Documentación, de la Facultad de Derecho, de la Facultad de Económicas y de la Facultad de Educación de las Universidades Complutense y Autónoma de Madrid y del Instituto Manuel Azaña de Getafe su colaboración desinteresada, pues con su participación han hecho posible la realización de este estudio.

2 "Las acciones pueden ser descritas en función de una estructura de bucle recursivo [...]" (N. del E.) 


\section{REFERENCIAS BIBLIOGRÁFICAS}

Alemany Martínez, D. \& Candela Hidalgo, A. R. (2011), "Pautas de comportamiento en la búsqueda de información en el entorno de la Universidad de Alicante: Acciones desde la docencia y los servicios bibliotecarios", en Teoría de la Educación. Educación y Cultura en la Sociedad de la Información, vol. 12, núm. 2, pp. 235-258.

Bates, M. J. (1989), "The design of browsing and berrypicking techniques for the online search interface", en Online Information Review, vol. 13, núm. 5, pp. 407-424.

_ (2010), "Information Behavior", en M. J. Bates \& M. N. Maack, (eds.), Encyclopedia of Library and Information Sciences, $3^{\mathrm{a}} \mathrm{ed}$., New York: CRC Press, vol. 3, pp. 2381-2391. Disponible en: http:// pages.gseis.ucla.edu/faculty/bates/articles/information-behavior. html (Fecha de consulta: 26/07/2013).

Bedny, G. Z. \& Harris, S. R. (2005), "The Systemic-Structural Theory of Activity: Applications to the Study of Human Work", en Mind, Culture, and Activity, vol. 12, núm. 2, pp. 128-147.

— ; Seglin, M. H. \& Meister, D. (2000), "Activity Theory: History, research and application”, en Theoretical Issues in Ergonomics Science, vol. 1, núm. 2, pp. 168-206.

Belkin, N. J. (1990), "The Cognitive Viewpoint in Information Science”, Journal of Information Science, vol. 16, núm. 1, pp. 11-15.

_ (1993), "Interaction with texts: Information retrieval as information seeking behavior", en Information retrieval '93: Von der Modellierung zur Anwendung, Konstanz: Universitaetsverlag Konstanz, pp. 55-66.

—; Oddy, R. N.; Brooks, H. M. (1982), "Ask for information Retrieval: Part I. Background and theory”, en Journal of Documentation, vol. 38, núm. 2, pp. 61-71.

British Library \& JISC (2008), "Information behaviour of the researcher of the future". Disponible en: http://www.jisc.ac.uk/media/docu ments/programmes/reppres/gg_final_keynote_11012008.pdf Existe traducción al español disponible en: http://revistas.um.es/anales doc/article/viewFile/24921/24221 (Fecha de consulta:26/07/2013).

Charmaz, K. (2006), Constructing Grounded Theory: A Practical Guide through qualitative analysis, London: SAGE.

Cole, C. (1997), "Information as process: The difference between corroborating evidence and 'information' in humanistic research domains", en Information Processing and Management, vol. 33, núm. 1, pp. 55-67.

Dervin, B. \& Nilan, M. (1986), "Information needs and uses", en Annual Review of Information Science and Technology, vol. 21, pp. 3-33.

Fidel, R.; Davies, R. K.; Douglass, M. H.; Holder, J. K. \& Hopkins, C. J. (1999), "A Visit to the Information Mall: Web Searching Behavior of High School Students", en Journal of the American Society for Information Science, vol. 50, núm. 1, pp. 24-37. 
Fisher, K. E.; Erdelez, S. \& McKechnie, L. (2005) (eds.), Theories of information behavior, Medford, NJ: Information Today.

Ge, X. (2010), "Information-Seeking Behavior in the Digital Age: A Multidisciplinary Study of Academic Researchers", en College E Research Libraries, vol. 71, núm. 5, pp. 435-455.

Glaser, B. G. \& Strauss, A. L. (1967), The discovery of Grounded Theory: Strategies for qualitative research, Chicago: Aldine Publishing.

Gómez Hernández, J. A. (2010), "Las bibliotecas universitarias y el desarrollo de las competencias informacionales en los profesores y los estudiantes", en Revista de Universidad y Sociedad del Conocimiento (RUSC), vol. 7, núm. 2, pp. 39-49. Disponible en: http:// rusc.uoc.edu/ojs/index.php/rusc/article/viewFile/v7n2-gomez/v7 n2-gomez (Fecha de consulta: 26/07/2013).

Gómez Restrepo, A. M. (2012), “Comportamiento en la búsqueda de información: el caso de los estudiantes de postgrado", en Revista Interamericana de Bibliotecología, vol. 35, núm. 2, pp. 133-148.

González-Teruel, A. (2005), Los estudios de necesidades y usos de información: fundamentos y perspectivas actuales, Gijón: Trea.

_ (2011), "La perspectiva del usuario y del sistema en la investigación sobre el comportamiento informacional", en Teoría de la Educación. Educación y Cultura en la Sociedad de la Información, vol. 12, núm. 1, pp. 9-27.

Ingwersen, P. (1982), "Search procedures in the library - analysed from the cognitive point of view", en Journal of Documentation, vol. 38, núm. 3, pp. 165-191.

- (2001), "Cognitive information retrieval", en Annual Review of Information Science and Technology, vol. 34, pp. 3-52.

Kim, J. (2007), "Modeling task-based information seeking on the web: Application of information seeking strategy schema", en Proceedings of the American Society for Information Science and Technology, vol. 44, pp. 1-13.

Kuhlthau, C. (1983), The library research process: Case studies and interventions with high school seniors in advanced placement english classes using Kelly's theory of constructs, D. Dissertation. Rutgers: The State University of New Jersey.

- (2004), Seeking meaning: A process approach to library and information services, $2^{a}$ ed., Westport, CT: Libraries Unlimited.

León, O. G. \& Montero, I. (1999), Diseño de investigaciones: Introducción a la lógica de la investigación en Psicología y Educación, Madrid: McGraw-Hill.

Nardi, B. M. (ed.) (1996), Context and consciousness: Activity Theory and Human-Computer Interaction, Cambridge, MA: MIT Press.

Salton, G. (1968), Automatic Information Organization and Retrieval, New York: McGraw-Hill.

Spink, A. \& Cole, C. (2005) (eds.), New Directions in Cognitive Information Retrieval, Dordrecht (The Netherlands): Springer. 
Strauss, A. \& Corbin, J. (1998), Basics of qualitative research: Techniques and procedures for developing grounded theory, 2a ed., Los Angeles, CA: Sage.

Thatcher, A. (2006), "Information-seeking behaviours and cognitive search strategies in different search tasks on the WWW", en International Journal of Industrial Ergonomics, vol. 36, pp. 1055-1068.

Valles, M. S. (1997), Técnicas cualitativas de investigación social. Reflexión metodológica y práctica profesional, Madrid: Síntesis.

Von Brevern, H. \& Synytsya, K. (2006), "A Systemic Activity based Approach for Holistic Learning \& Training Systems", en Educational Technology \& Society, vol. 9, núm. 3, pp. 100-111.

Wilson, T. D. (1981), "On user studies and information needs", en Journal of Documentation, vol. 37, pp. 3-15. Disponible en: http:// s3.amazonaws.com/academia.edu.documents/30542309/on_user _studies_and_information_needs.pdf (Fecha de consulta: 26/07/2013).

_ (1997), "Information Behavior: An Interdisciplinary Perspective", en Information Processing and Management, vol. 33, núm. 4, pp. 551-572.

_ (1999), "Models in information behavior research", en Journal of Documentation, vol. 55, núm. 3, pp. 249-270. Disponible en: http://informationr.net/tdw/publ/papers/1999JDoc.html (Fecha de consulta: 26/07/2013).

_ (2000), "Human Information Behavior", en Information Science (Número especial sobre Information Science Research), vol. 3, núm. 2, pp. 49-55. Disponible en: http://inform.nu/Articles/ Vol3/v3n2p49-56.pdf (Fecha de consulta: 26/07/2013).

Zipf, G. K. (1949), Human Behavior and the Principle of Least Effort, Cambridge, MA: Addison-Wesley. 
Anexo

Guión de la entrevista en profundidad

Tarea 1. Clasificación

1. Pasos seguidos en la realización de la tarea.

2. Motivos de la elección de las diversas categorías principales analizadas.

3. Causas del orden de las categorías analizadas.

4. Razones de la profundización o no en las categorías analizadas.

5. Pensamientos al no localizar el tema en las categorías analizadas.

6. Reacciones ante las dudas 0 la frustración.

7. Posibles modificaciones en la clasificación del tema propuesto.

Tarea 2. Concepto matemático y cálculo elemental

1. Pasos seguidos en la realización de la tarea.

2. Pensamientos al tener que emplear el concepto de número primo.

3. Procedimiento seguido para superar el desconocimiento del concepto.

4. Motivos para la elección de dicho procedimiento.

5. Reacciones ante la necesidad informativa no satisfecha. Búsqueda de alternativas.

6. Procedimiento seguido para descubrir los números que cumplen esa condición.

7. Motivos para la elección de dicho procedimiento.

8. Reacciones ante las dudas.

9. Procedimiento o procedimientos habitualmente seguidos para la realización de las operaciones matemáticas elementales.

10. Razones de la elección del procedimiento seguido para el cálculo.

11. Sugerencias de asistencia al usuario en tales circunstancias.

Tarea 3. Memorización

1. Pasos seguidos en la realización de la tarea.

2. Opinión sobre las respectivas temáticas de los textos.

3. Reacciones al tener que memorizar textos de dichas temáticas.

4. Motivos para la consideración del grado de dificultad de los textos.

5. Influencia de la dificultad del texto en la tarea de memorización.

6. Influencia de la extensión del texto en la tarea de memorización.

7. Razones de la selección de datos a memorizar.

8. Influencia del tipo de datos (palabras, cifras, siglas...) en la tarea de memorización.

9. Método o métodos de memorización de los datos seleccionados.

10. Motivos para la elección del método o métodos de memorización.

Tarea 4. Categorización

1. Pasos seguidos en la realización de la tarea.

2. Criterios utilizados para la identificación de la temática de las páginas.

3. Causas de la dificultad en la identificación de la temática de las páginas.

4. Reacciones ante la dificultad en la identificación temática. Búsqueda de alternativas.

5. Motivos para la designación de una categoría de entre varias posibles cuando la temática de una página lo permite.

6. Reacciones ante las dudas en la adscripción de páginas a las categorías.

7. Posibles modificaciones en el diseño de las páginas web para facilitar la identificación de la temática. 